\title{
A Repetitive Control Scheme for Industrial Robots Based on B-Spline Trajectories
}

\author{
Luigi Biagiotti, Lorenzo Moriello, Claudio Melchiorri
}

\begin{abstract}
In this paper, a novel repetitive control scheme is presented and discussed. The general framework is the control of repetitive tasks of robotic systems or, more in general, of automatic machines. The key idea of the proposed scheme consists in modifying the reference trajectory provided to the plant in order to compensate for external loads or unmodelled dynamics that cyclically affect it. By exploiting the dynamic filters for the B-spline trajectory planning, it has been possible to integrate the trajectory generation within a repetitive control scheme able to modify in real-time the reference signal with the aims of nullify interpolation errors. Experimental results obtained controlling two joints of a standard industrial manipulator are reported, showing the effectiveness of the proposed method.
\end{abstract}

\section{INTRODUCTION}

In practical applications, desired tasks are often repetitive or cyclic in nature. This is particularly true in industrial robotics and in automatic machines, where many tasks simply imply the continuous repetition of a given motion. From a control point of view, it is therefore required to track and/or reject a periodic exogenous signal that can be considered known since it refers to planned trajectories or disturbances whose cycle time is easily measurable or known in advance. In order to improve the tracking accuracy, Repetitive Control (RC) represents a simple and effective method, since it aims at cancelling tracking errors over repetitions by learning from previous iterations. RC was first developed by Inoue et al. [1], [2] to improve the control of the power supply in a proton synchrotron accelerator, but soon was applied to many other different systems. Many surveys, see e.g. [3], [4], report the successful use of RC in a number of applications, such as high accuracy trajectory tracking of servomechanism, torque vibration suppression in motors, noise cancellation in power supply, industrial robotics, and so on.

In this paper, a novel repetitive control scheme is presented. The scheme is based on a proper modification of the reference trajectory for the plant, which is supposed to be already controlled. A similar idea has been already proposed in the continuous-time domain in [5], where a twodegree-of-freedom local controller, and a plug-in type RC is used to update the reference trajectory. In our approach, we

L. Biagiotti is with the Department of Engineering "Enzo Ferrari", University of Modena and Reggio Emilia, via Pietro Vivarelli 10, 41125 Modena, Italy, e-mail: luigi.biagiotti@unimore.it.

C. Melchiorri and L. Moriello are with the Department of Electrical, Electronic and Information Engineering "Guglielmo Marconi", University of Bologna, Viale del Risorgimento 2, 40136 Bologna, Italy, e-mail: \{claudio.melchiorri\},\{lorenzo.moriello2\}@unibo.it.

This activity has been supported by the University of Bologna, with the FARB Linea 2 funding action. assume that the reference trajectories are defined by spline functions, which are de-facto the standard tool used in the industrial field for planning complex motions interpolating a set of given via-points [6]. Thanks to the possibility of generating B-spline trajectories by means of dynamic filters [7], the trajectory planner has been inserted in an external feedback control loop that modifies in real-time the control points of the B-spline curve so that the tracking error at the desired via-points converges to zero. The proposed control scheme has been directly developed in the discrete timedomain, and is characterized by a very low computational complexity. Moreover, the application of this control scheme is independent of the particular control law of the plant, making it suitable to enhance the performance of inaccessible industrial controllers. In particular, in Sec. II a general overview of the filters for B-spline generation is given both in the continuous- and in the discrete-time domain. Then in Sec. III the repetitive control scheme based on B-spline filters is illustrated and its stability is analyzed. Experimental results are reported in Sec. IV, while final conclusions are discussed in Sec. V.

\section{SET-POINT GENERATION VIA B-SPLINE FILTERS}

In a number of practical applications the reference signal for dynamical systems is defined by using spline functions that interpolate a set of desired via-points $q_{i}^{\star}, i=0, \ldots, n-$ 1 at time instants $t_{i}$. By assuming a B-spline form of the trajectory, i.e.

$$
q(t)=\sum_{i=0}^{n-1} p_{i} B_{i}^{d}(t), \quad t_{0} \leq t \leq t_{n-1}
$$

where $B_{i}^{d}(t)$ is a B-spline basis function of degree $d$, the control points $p_{i}$, which determine the shape of the curve, must be computed by imposing interpolation conditions on the given data points $q_{i}^{\star}$, see [6]. Then, the spline $q(t)$ can be determined $\forall t \in\left[t_{0}, t_{n-1}\right]$ by computing the basis functions $B_{i}^{d}(t)$ via numerical procedures usually based on recursion. If uniform B-splines are considered, i.e. B-splines characterized by an equally-spaced distribution of the knots $t_{i}$ i.e. $t_{i+1}-t_{i}=T, i=0, \ldots n-2$, the generation of the trajectory can be obtained by means of a chain of $d$ dynamic filters defined as

$$
M(s)=\frac{1-e^{-s T}}{T s}
$$

fed by the staircase signal $p(t)$ obtained by maintaining the value of each control point $p_{i}$ for the entire period $i T \leq$ $t<(i+1) T$. See the scheme of Fig. 1 and the signals 


\begin{tabular}{l}
\hline$F_{1}\left(z^{-1}\right)=z^{-1}$ \\
$F_{2}\left(z^{-1}\right)=\frac{1}{2} z^{-1}+\frac{1}{2} z^{-2}$ \\
$F_{3}\left(z^{-1}\right)=\frac{1}{6} z^{-1}+\frac{4}{6} z^{-2}+\frac{1}{6} z^{-3}$ \\
$F_{4}\left(z^{-1}\right)=\frac{1}{24} z^{-1}+\frac{11}{24} z^{-2}+\frac{11}{24} z^{-3}+\frac{1}{24} z^{-4}$ \\
$F_{5}\left(z^{-1}\right)=\frac{1}{120} z^{-1}+\frac{26}{120} z^{-2}+\frac{66}{120} z^{-3}+\frac{26}{120} z^{-4}+\frac{1}{120} z^{-5}$ \\
\hline
\end{tabular}

TABLE I

EXPRESSION OF THE FILTER $F_{d}\left(z^{-1}\right)$ FOR DIFFERENT VALUES OF $d$.

shown in Fig. 2, where the generation of a cubic B-spline is considered. Note that $p(t)$ is obtained by applying a zeroorder hold to the train of impulses of amplitude $p_{i}$. Moreover, it is worth noticing that the output trajectory is delayed with respect to the application of control points of $m T$ seconds, where $m=\frac{d+1}{2}$.

For computer controlled systems equipped with digital controllers with sampling period $T_{s}$, the B-spline reference trajectory can be computed at time-instants $k T_{s}$ by discretizing the filter of Fig. 1. By Z-transforming the chain of $d$ filters $M(s)$ with zero-order hold the system of Fig. 3 is obtained, where $F_{d}\left(z^{-1}\right)$ is a FIR filter whose expression is reported in Tab. I for $d=1, \ldots, 5$.

In this case, the sequence $p_{i}$ of the control points is transformed in the staircase sequence $p_{k}$, with sampling time $T_{s}$, by means of an upsampling operation with replication

$$
p_{k}=p_{i}, \quad k=i N+j, \quad j=0, \ldots, N-1, \forall i
$$

where $N$ denotes the ratio, supposed to be an integer, between $T$ and $T_{s}$. The samples of the B-spline sequence are then generated by the filter denoted by $M_{d}(z)$ and coincide with the value of the continuous-time trajectory at time instants $k T$, i.e $q_{k}=q(k T)$, see Fig. 4 .

The reference trajectory generated by the discrete B-spline filter is then provided to the plant, as illustrated in Fig. 5. Since this scheme has a standard cascade structure without control actions but with the only purpose of generating arbitrarily complex trajectories for the plant $G(z)$, the capabilities of $G(z)$ to track such a kind of signals are implicitly assumed. Therefore, the system $G(z)$ is assumed to be a controlled plant, with a standard closed-loop structure, whose frequency response is characterized by a typical low-pass behavior with a static gain as close as possible to the unity. In order to follow the input signal accurately, the bandwidth of system must be large enough [8], and in particular larger than the maximum spectral components of the input. In case of uniform B-splines generated by the linear filter $M_{d}(z)$, the spectrum of the resulting trajectories can be determined by analyzing the frequency response of $M_{d}(z)$. In particular the magnitude of $M_{d}\left(e^{j \omega T_{s}}\right)$ is

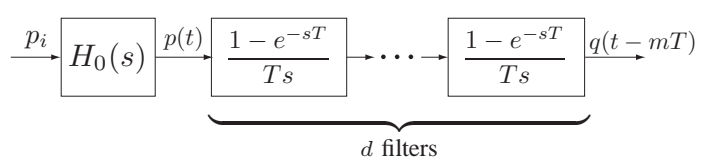

Fig. 1. System composed by $d$ mean filters and by a zero-order hold $H_{0}(s)$ for the computation of continuous-time B-spline trajectories of degree $d$.
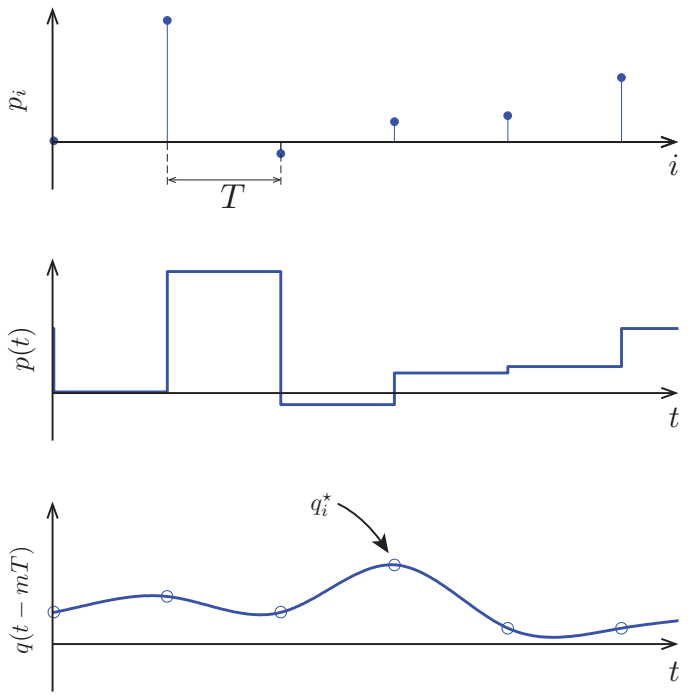

Fig. 2. Control points sequence $p_{i}$ defining a cubic B-spline and related reference trajectory $q\left((k-m N) T_{s}\right)$ with $m=2$ obtained with the dynamic filter of Fig. 3.

$$
\left|M_{d}\left(e^{j \omega T_{s}}\right)\right|=\left|F_{d}\left(e^{-j \omega}\right)\right| \cdot\left|\frac{\operatorname{sinc}\left(\frac{\omega}{\omega_{0}}\right)}{\operatorname{sinc}\left(\frac{\omega}{\omega_{s}}\right)}\right|^{d}, \quad \omega \leq \frac{\omega_{s}}{2}
$$

where $\operatorname{sinc}(\cdot)$ denotes the normalized sinc function defined as $\operatorname{sinc}(x)=\frac{\sin (\pi x)}{\pi x}$ and $\omega_{0}=\frac{2 \pi}{T}, \omega_{s}=\frac{2 \pi}{T_{s}}$. The FIR filter $F_{d}\left(e^{-j \omega}\right)$ has a standard low-pass behavior, therefore $\left|M_{d}\left(e^{j \omega T_{s}}\right)\right|$ is a low-pass filter as well, and its magnitude decreases rather quickly as $\omega$ grows, especially for high values of $d$. In Fig. 6, the frequency response of the cubic $(d=3)$ B-spline filter is reported. Obviously, the frequency response of $M_{d}(z)$ is a good approximation of that of the continuous time generator (the approximation level depends on the ratio $N$ between $T$ and $T_{s}$ ). Fig. 6 highlights that spectrum components of the reference trajectory $q^{r}(t-m T)$ at the output of this filter are significant only in the frequency range $\left[0, \omega_{0}\right]$, while the reduction of the components for $\omega>\omega_{0}$ is at least of two order of magnitude $(-40 \mathrm{db})$.

From common practice, it is known that in order to obtain good tracking performances, the controlled plant $G(z)$ must have a cutoff frequency $\omega_{c} \gg \omega_{0}$ (typical values are $\omega_{c} \geq$ $\alpha \omega_{0}$, with $\alpha=5 \div 10$ ). Consequently, since the sampling frequency $\omega_{s}$ is chosen as $\omega_{s} \geq \beta \omega_{c}$ with $\beta=5 \div 10$, the minimum value of $N$ may range between 25 and 100 .

Unfortunately, even if the considerations on $G(z)$ above mentioned are verified, that is

$$
G\left(e^{j \omega T_{s}}\right) \approx 1 \text { for } \omega \leq \frac{2 \pi}{T} \ll \omega_{c}
$$

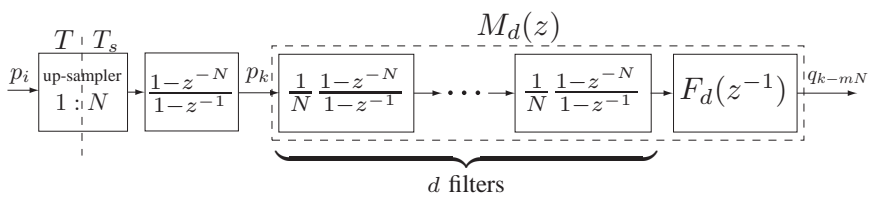

Fig. 3. System $M_{d}(z)$ composed by $d$ moving average filters and by the FIR filter $F_{d}\left(z^{-1}\right)$ defined in (I) for the generation of discrete-time B-spline trajectories of degree $d$. 

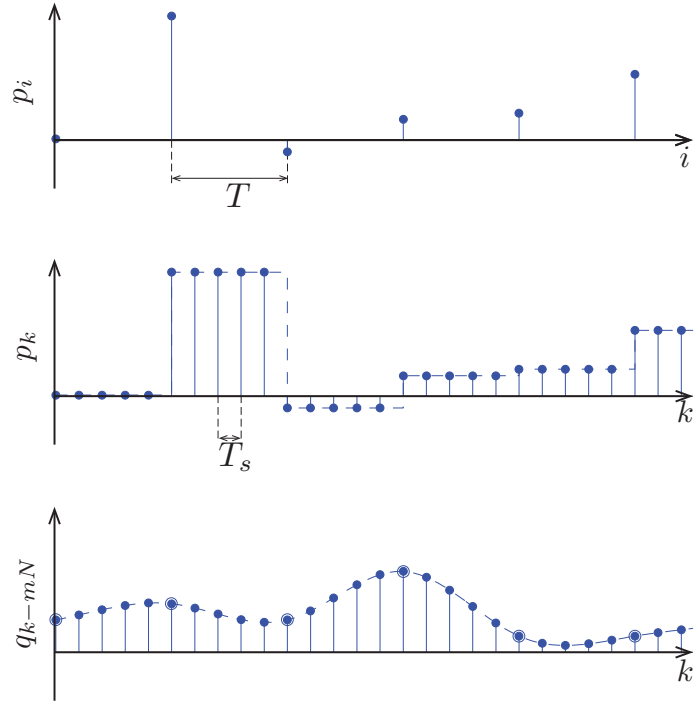

Fig. 4. Control points sequence $p_{i}$ defining a cubic B-spline and related reference trajectory $q_{k-m N}$ with $m=2$ obtained with the dynamic filter of Fig. 3.

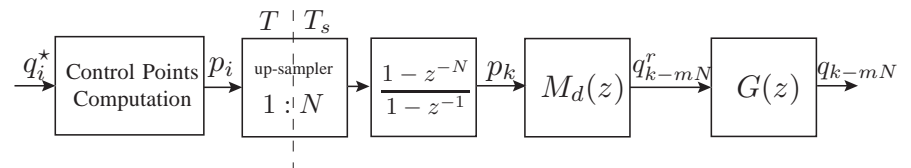

Fig. 5. Set-point definition by means of B-spline filter for a (controlled) discrete-time system $G(z)$.

the tracking error $e=q-q^{r}$ between plant output and reference B-spline trajectory can be relevant, because $G\left(e^{j \omega T}\right)$ is equal to one only approximatively and may be affected by external disturbances.

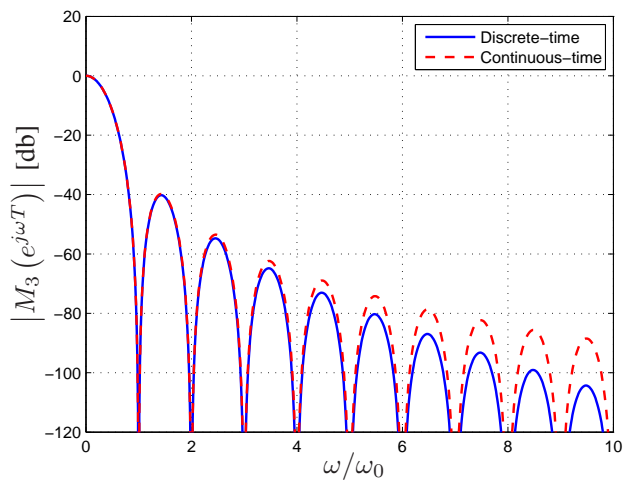

Fig. 6. Magnitude of the frequency response of the B-spline filter $M_{d}(z)$ for $d=3$ (and $N=25$ ) compared with the frequency response of the continuous-time generator $M_{d}(s)$.

\section{REPETITIVE CONTROL BASED ON DISCRETE-TIME B-SPLINE FILTER GENERATOR}

We assume here that the tasks to be performed are cyclic, and therefore that the trajectories to be tracked are repetitive. Moreover, we assume that also "external" disturbances have the same property, i.e. that either external loads or unmodeled dynamics depend on the current state of the system. In this case, it is possible to implement a procedure for modifying the reference signal in order to guarantee that the interpolation error at the given via-points $q_{i}^{\star}$ asymptotically vanishes. Since a B-spline curve is completely determined by the position of its control points, the modification of the trajectory is obtained by directly acting on them. In any case, it is necessary to determine initially the value of the control points called $p_{i}^{\star}$ that define the $\mathrm{B}$-spline passing through the via-points $q_{i}^{\star}$ at time instants $t_{i}=i T$. Being the trajectory periodic, the so-called periodic splines should be adopted, i.e. spline functions for which

$$
q^{(j)}\left(t_{0}\right)=q^{(j)}\left(t_{n}-1\right), \quad j=1, \ldots, d-1
$$

where $d$ is the degree of the spline. If uniform cubic Bsplines are considered, the interpolation conditions $q(i T)=$ $q_{i}^{\star}$, which can be written as

$$
q(i T)=\frac{1}{6} p_{i-1}^{\star}+\frac{4}{6} p_{i}^{\star}+\frac{1}{6} p_{i+1}^{\star}=q_{i}^{\star}, \quad i=0, \ldots, n-1
$$

and the additional constraints (4) lead to the linear system

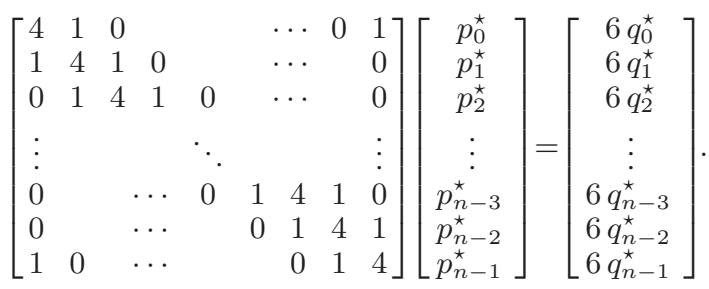

whose solution provides the sequence of control points $p_{i}^{\star}$. Note that these control points do not depend on the particular choice of the time $T$ defining the interpolation time-instants. The scheme of Fig. 7 shows the mechanism for B-spline modification based on the RC approach. In this scheme, both the trajectory generator and the plant $G(z)$ are inserted in a discrete-time control loop that, on the basis of the interpolation error $\tilde{q}_{i}=q_{i}^{\star}-q_{i}$, modifies in real-time the control points sequence (denoted by $p_{i}^{r}$ ) from the initial value $p_{i}^{\star}$. It is a typical dual rate system with the feedback loop running at a sampling time $T$ considerably higher than the period $T_{s}$ of the trajectory generator and of the controlled plant $G(z)$.

The system $H(z)$ is a FIR filter that approximates the relation between via-points and control points in (5), i.e.

$$
\frac{P(z)}{Q(z)}=\frac{6}{z+4+z^{-1}}
$$

for cubic B-spline. Note that (7) represents an unstable system and consequently it cannot be used for computing the sequence $p_{i}$ from $q_{i}$. This is a direct consequence of the fact that the interpolation procedure is a global problem that involves all the via-points $q_{i}$. However, it is possible to approximate the interpolation process by taking into account only a small set of points $q_{i}$. This approach leads to a FIR filter defined by

$$
H(z)=\sum_{n=-r}^{r} h(n) z^{-n}
$$

that approximates the impulse response of (7) within a prescribed tolerance according to the value of $r$. The coefficients $h(n)$ for $d=3$ can be computed as 


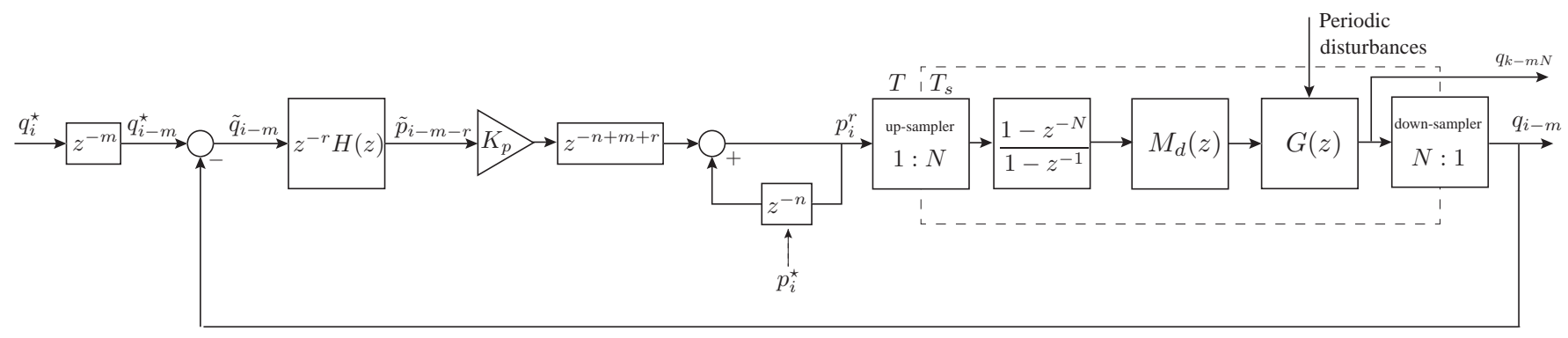

Fig. 7. Discrete-time repetitive control scheme based on discrete-time B-spline filter.

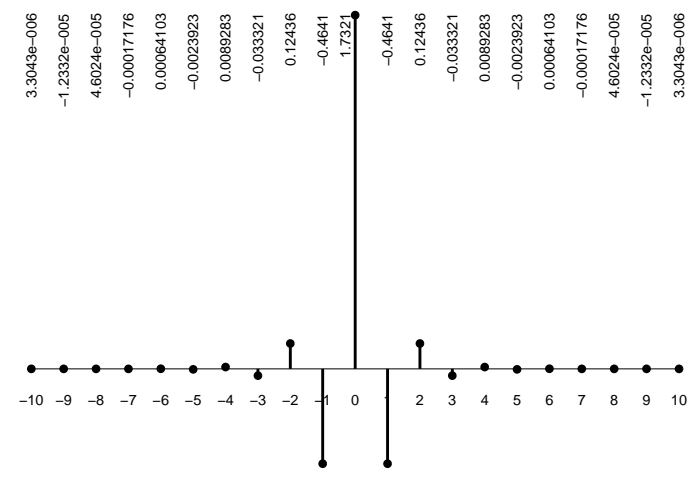

Fig. 8. Impulse response $h(n)$ of the filter (7).

$$
h(n)=\frac{1-\alpha}{1+\alpha} \alpha^{|n|}
$$

where $\alpha=-2+\sqrt{3}$ is the stable pole of (7). The sequence $h(n)$ is shown in Fig. 8. Note that the value of $h(n)$ becomes extremely small as $|n|$ grows. (for more details see [9]). The quality of the approximation depends on the order $2 r+1$ of the filter, but it is worth noticing that the choice $r=4$ guarantees an approximation error with respect to the exact solution of (6) smaller than $0.5 \%$. In the scheme of Fig. 7 , the filter $H(z)$ is used to transform the interpolation errors $\tilde{q}_{i}$ in an error in the control points position $\tilde{p}_{i}$. Since $H(z)$ is not a causal filter, it is necessary to introduce a delay equal to $r$ to make it feasible, that is

$$
H^{\prime}(z)=z^{-r} H(z)=\sum_{n=0}^{2 r} h(n-r) z^{-n} .
$$

The sequence $\tilde{p}_{i}$ multiplied by the constant $K_{p}$, assumed to be equal to one, and properly delayed in time is provided to the filter

$$
\frac{1}{1+z^{-n}}
$$

used to compute the reference sequence of points $p_{i}^{r}$ for the discrete-time interpolator based on B-splines and the controlled plant. Note that the initial value of the output of filter in (10) has been set to $p_{i}^{\star}$, that is the sequence of the control points defining the ideal trajectory.

Theorem 1: The control scheme of Fig. 7, subject to periodic disturbances, guarantees that the interpolation error $\tilde{q}_{i}=q_{i}^{\star}-q_{i}$ between the desired via-points and the plant output at time $t_{i}=i T=k N T_{s}$ asymptotically converges to zero provided that the plant $G(z)$ meets the trajectory tracking condition (3).

Proof: According to the theory of discrete-time repetitive control [10], that exploits the internal model principle [11], the presence in the control loop of the transfer function (10) assures asymptotic perfect tracking of a periodic signal with period $n$ (in this case the sequence of desired via points $\left.q_{i}^{\star}\right)$ if the stability of the whole system is assured.

Because of the structure of the control scheme, the stability analysis of the system at the slow sampling rate $(T)$ can be deduced by neutralizing the effects of up-sampler and downsampler. By considering the cascade of the filter $H(z)$ and of the system with period $T_{s}$ composed by plant and trajectory generator, as shown in Fig. 9(a), it is possible to simplify the scheme by means of some formal manipulations:

- Since the controlled plant $G(z)$ is supposed to have a standard low-pass structure, in a worst case perspective, in lieu of the transfer function $G(z)$ the (constant) complex number

$$
G_{\mathrm{wc}}=\max _{\omega \leq \omega_{0}}\left|G\left(e^{j \omega T_{s}}\right)\right| \mathrm{e}^{j \min _{\omega \leq \omega_{0}}\left\{\arg G\left(e^{j \omega T_{s}}\right)\right\}}
$$

can be considered in order to take into account the maximum gain variation and the maximum (negative) phase displacement caused by $G(z)$. The use of the Bspline filter allows to restrict the range of variation of $\omega$ to the interval $\left[0, \omega_{0}\right]$ because, as already noted, the reference signal for the plant can be considered null outside this interval. In this way, the block describing the plant and the down-sampler can be exchanged, as shown in Fig. 9(b).

- the filter $H(z)$ which approximates the relation between via-points $q_{i}^{\star}$ and control points $\hat{p}_{i}^{\star}$ is followed by the B-Spline generator which, fed by the control points $\hat{p}_{i}^{\star}$, provides at knots $i T$ the desired via-points $\hat{q}_{i-m}^{\star}$ delayed of $m T$ instants ${ }^{1}$. As consequence this cascade can be reduced to a simple time-delay $z^{-m}$, as shown in Fig. 9(c).

Finally, the scheme of Fig. 7 can be reduced to the one shown in Fig. 10, that runs with a sampling period $T$. It is a

\footnotetext{
${ }^{1}$ Note that $\hat{p}_{i}^{\star}$, and consequently $\hat{q}_{i-m}^{\star}$, is only an approximation of the real value $p_{i}^{\star}$, because of the filter $H(z)$. However, the level of the approximation can be arbitrarily improved by assuming larger values of $r$. If the interpolation of $n$ via-points with B-spline trajectory of degree $d$ is considered, the optimal (highest) value of $r$ is $r=n-m$, being $m=\frac{d+1}{2}$.
} 


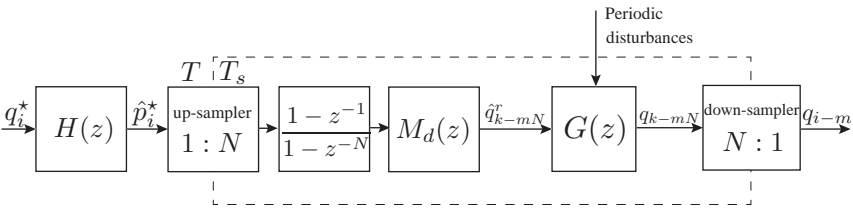

(a)

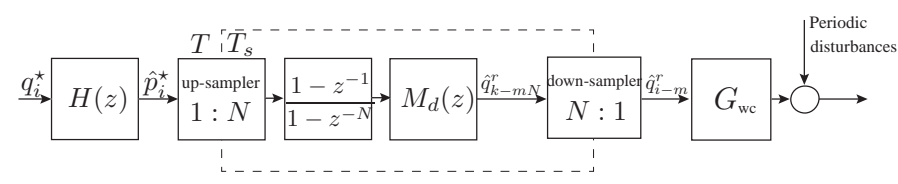

(b)

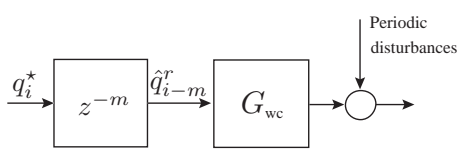

(c)

Fig. 9. Model reduction of the discrete-time repetitive control scheme based on B-spline filter.

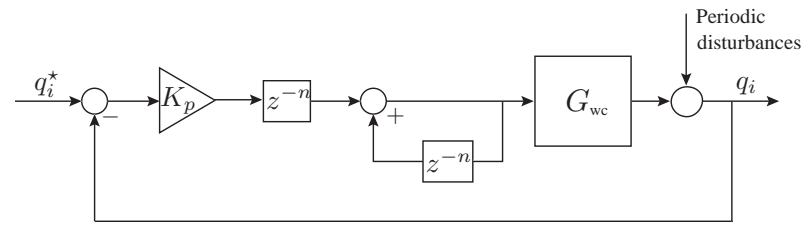

Fig. 10. Discrete-time repetitive control scheme with sampling period $T$ obtained from the dual-rate scheme of Fig. 7 based on discrete-time B-spline filter.

quite standard repetitive control scheme whose stability can be inferred by analyzing its characteristic equation

$$
1+\frac{z^{-n}}{1-z^{-n}} K_{p} G_{\mathrm{wc}}=0
$$

By following the approach proposed in [12], it is possible to see that the asymptotic stability of (11) is equivalent to the stability of the feedback system with loop-transfer function

$$
L(z)=z^{-n}\left(K_{p} G_{\mathrm{wc}}-1\right) .
$$

Therefore, by applying the Nyquist criterion it descends that all the poles of (11) are within the unit circle if and only if the polar plot of $L\left(e^{j \omega T}\right)$ for $-\frac{\pi}{T} \leq \omega \leq \frac{\pi}{T}$ does not encircle or touch the critical points -1 . This can be assured by imposing that

$$
\left\|K_{p} G_{\mathrm{wc}}-1\right\|<1 .
$$

Being $K_{p} \leq 1$ (usually $K_{p}=1$ ), the stability condition (12) holds if condition (3) is met (in this case $G_{\mathrm{wc}} \approx 1$ ).

\section{EXPERIMENTAL EVAlUATION ON COMAU SMART5 SIX INDUSTRIAL MANIPULATOR}

In order to experimentally evaluate the proposed method the setup of Fig. 11 has been arranged. The system is composed of a COMAU Smart5 Six industrial robotic arm, a COMAU C4G Controller and a standard PC with an Intel Core 2 Duo $2.4 \mathrm{GHz}$ processor and $1 \mathrm{~GB}$ of RAM. The COMAU Smart5 Six is a 6 DOF robot with anthropomorphic structure, with a payload of $6 \mathrm{Kg}$. The robot is driven

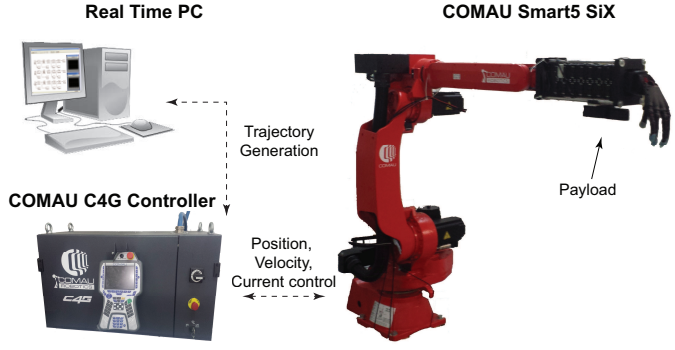

Fig. 11. Experimental setup.

by the COMAU C4G Controller that performs both the position/velocity control (adaptive control) and the power stage management with current control of each joint. The C4G Controller also implements a software option called "C4G OPEN" that allows the integration of the robot control unit with the external personal computer, in order to develop complex control systems at high hierarchical level. The C4G Open architecture is based on a real time communication on Ethernet network between the controller and the real time PC. In particular the PC runs on the real-time operating system RTAI-Linux on a Ubuntu NATTY distribution with Linux kernel 2.6.38.8 and RTAI 3.9 that allows the trajectory generator to run with a sampling period $T_{s}=1 \mathrm{~ms}$.

For the design of the control scheme and of trajectory generator, the MatLab/Simulink/RealTime Workshop environment has been used.

For sake of simplicity, the RC of Fig. 7 has been implemented on the third joint only, while the second joint has been actuated in order to disturb the third joint because of the dynamic coupling. Obviously, both joints are required to track two different cyclic spline trajectories with the same period. In particular, each trajectory interpolates 12 viapoints $q_{i}^{\star}$ with uniform knot span $T=1 s$. In Fig. 12 the performance of the system is shown. As can be seen, the third

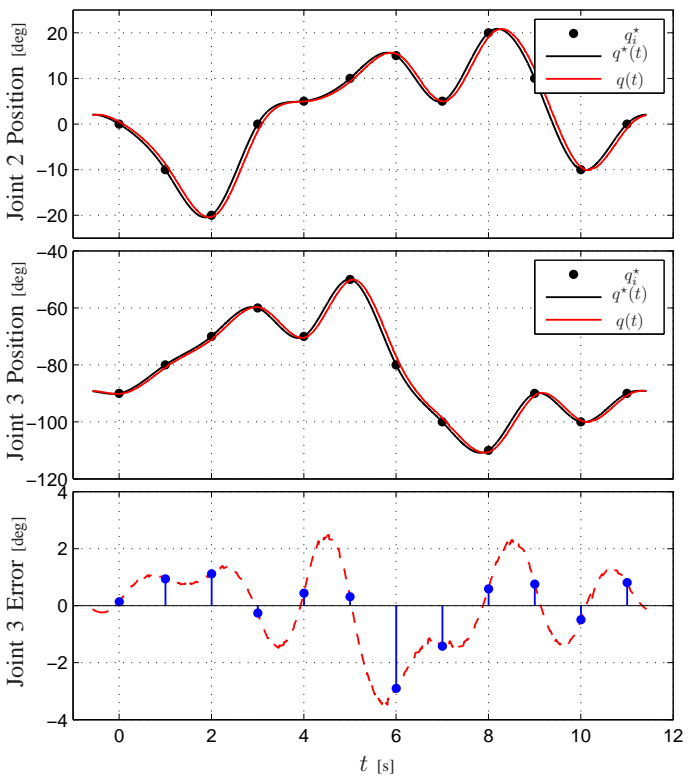

Fig. 12. Tracking performance of the system with the factory controller (without RC). 


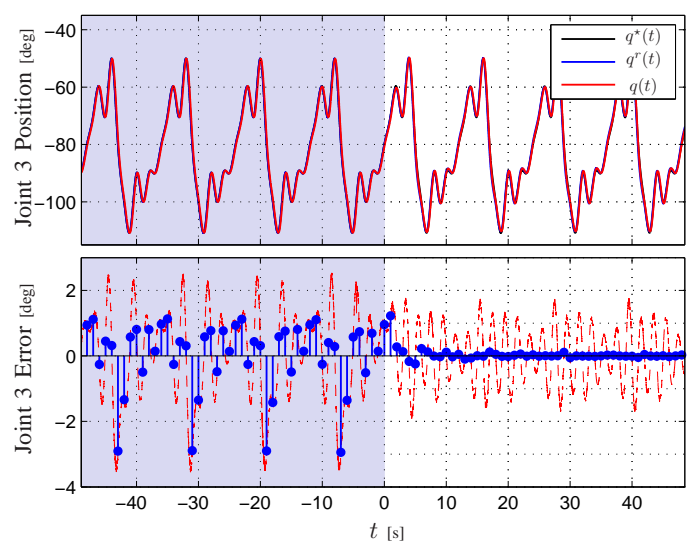

Fig. 13. Response of the system at the activation of the Repetitive Control $(t=0)$. Errors at sampling instants $T$ are highlighted in blue.

joint is affected by a quite evident tracking error, due to both the second joint movement and a $3 \mathrm{Kg}$ payload represented by the UBHand IV robotic hand [13]. It is worth noting that the tracking error is relevant even in correspondence of the points $q_{i}^{\star}$ that define the spline trajectory.

In Fig. 13 the tracking performance of the third joint is presented when the RC is switched on. It can be noted that the error in correspondence of the via-points $q_{i}^{\star}$ drastically decays in a few cycles.

In Fig. 14 a detail of the trajectory tracking with RC (after 5 cycles) is shown: in this case the reference trajectory $q^{r}(t)$ is different from the theoretical spline $q^{\star}(t)$, as it is modified by the controller in order to suppress tracking error at instants T. By comparing Fig. 12 and Fig. 14 the reduction of the tracking error is evident, particularly in correspondence of the points $q_{i}^{\star}$.

\section{CONCLUSIONS}

In this paper, motion planning and reactive control have been integrated in order to obtain a perfect tracking of a desired set of via-points. By considering cyclic tasks, which are quite common in the industrial and robotics field, a trajectory generation based on B-spline has been enhanced with a RC-type mechanism that modifies in real-time the control points defining the spline in order to nullify the tracking error at the desired points. The effectiveness of the proposed approach has been demonstrated both analytically and experimentally. In particular, tests performed on an industrial manipulator have shown that this scheme can be used to enhance the performance of the original position controller of the robot. Finally, the proposed approach could be used to refine the computation of the control points for a given motion trajectory in order to compensate for cyclic disturbances that characterize the plant. After an initial "training" the modified control points $p_{i}^{r}$ that take into account the dynamic behavior of the plant could be used without the adaptation mechanism in lieu of the theoretical values $p_{i}^{\star}$.

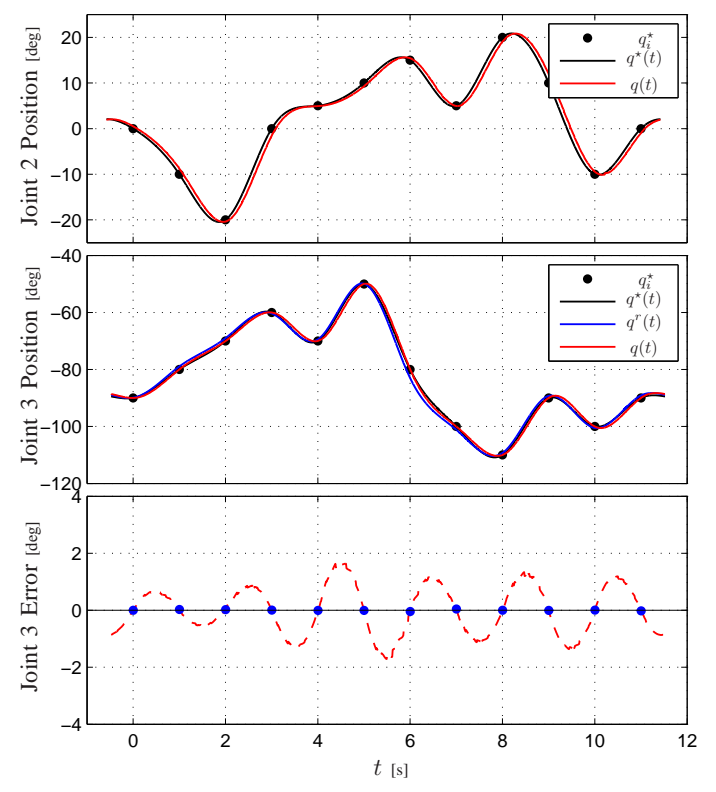

Fig. 14. Tracking performance of the system with RC controller activated. In the middle, the modified reference trajectory $q^{r}(t)$ for the third joint is reported in blue, as a result of the implementation of the $\mathrm{RC}$ controller.

\section{REFERENCES}

[1] T. Inoue, S. Iwai, and M. Nakano, "High accuracy control of a proton synchrotron magnet power supply," in Proc. 8th IFAC World Congress, vol. 20, 1981, pp. 3137-3142.

[2] T. Inoue, M. Nakano, and S. Iwai, "High accuracy control of servomechanism for repeated contouring," in Proc. 10th Annual Symp. Incremental Motion Contr. Syst. and Device, 1981, pp. 258-292.

[3] L. Cuiyan, Z. Dongchun, and Z. Xianyi, "A survey of repetitive control," in Intelligent Robots and Systems, 2004. (IROS 2004). Proceedings. 2004 IEEE/RSJ International Conference on, vol. 2, Sept 2004, pp. 1160-1166 vol.2.

[4] Y. Wang, F. Gao, and F. J. D. III, "Survey on iterative learning control, repetitive control, and run-to-run control," Journal of Process Control, vol. 19, no. 10, pp. 1589 - 1600, 2009. [Online]. Available: http://www.sciencedirect.com/science/article/pii/S0959152409001681

[5] S. Hara, Y. Yamamoto, T. Omata, and M. Nakano, "Repetitive control system: a new type servo system for periodic exogenous signals," Automatic Control, IEEE Transactions on, vol. 33, no. 7, pp. 659668, Jul 1988.

[6] L. Biagiotti and C. Melchiorri, Trajectory Planning for Automatic Machines and Robots, 1st ed. Heidelberg, Germany: Springer, 2008.

[7] — , "B-spline based filters for multi-point trajectories planning," in Robotics and Automation (ICRA), 2010 IEEE International Conference on, May 2010, pp. 3065-3070.

[8] K. Ogata, Modern control engineering (3rd ed.). Upper Saddle River, NJ, USA: Prentice-Hall, Inc., 1997.

[9] L. Biagiotti and C. Melchiorri, "Online trajectory planning and filtering for robotic applications via b-spline smoothing filters," in Intelligent Robots and Systems (IROS), 2013 IEEE/RSJ International Conference on, Nov 2013, pp. 5668-5673.

[10] M. Tsai, G. Anwar, and M. Tomizuka, "Discrete time repetitive control for robot manipulators," in Robotics and Automation, 1988. Proceedings., 1988 IEEE International Conference on, Apr 1988, pp. 1341-1346 vol.3.

[11] B. Francis and W. Wonham, "The internal model principle for linear multivariable regulators," Applied Mathematics and Optimization, vol. 2, no. 2, pp. 170-194, 1975. [Online]. Available: http://dx.doi.org/10.1007/BF01447855

[12] M. Tomizuka, T.-C. Tsao, and K.-K. Chew, "Discrete-time domain analysis and synthesis of repetitive controllers," in American Control Conference, 1988, June 1988, pp. 860-866.

[13] C. Melchiorri, G. Palli, G. Berselli, and G. Vassura, "Development of the ub hand iv: Overview of design solutions and enabling technologies," Robotics Automation Magazine, IEEE, vol. 20, no. 3, pp. 72-81, Sept 2013. 